\title{
SINERGIA
}

REVISTA DO INSTITUTO DE CIÊNCIAS ECONÔMICAS, ADMINISTRATIVAS E CONTÁBEIS (ICEAC)

\section{ECONOMIA CIRCULAR E BIOECONOMIA: UM NOVO CAMINHO PARA A SUSTENTABILIDADE?}

\author{
EMILIA DE OLIVEIRA FARIA \\ ARMANDO DE AZEVEDO CALDEIRA PIRES
}

\begin{abstract}
RESUMO
Diante do consenso sobre a urgência das questões ambientais e a crescente preocupação sobre a escassez dos recursos naturais, novos modelos de desenvolvimento têm sido propostos. Considerando a contribuição potencial das abordagens de Economia Circular (EC) e Bioeconomia para esse contexto, este estudo buscou responder a seguinte questão de pesquisa: como os modelos de EC e Bioeconomia se relacionam na literatura? A partir dessa indagação foi desenvolvida uma revisão sistemática da literatura com o objetivo de analisar: (i) como as pesquisas abordam essa relação; (ii) quais são as características desses estudos; (iii) quais os aspectos metodológicos mais recorrentes; (iv) quais são as principais iniciativas e programas existentes; e (v) quais são os principais atores. Este estudo permitiu avançar no conhecimento sobre a EC e a Bioeconomia na medida em que os principais resultados demonstraram o aumento do interesse dos pesquisadores na temática, apontaram para a complementaridade das abordagens a partir da rede de palavras-chave gerada, elencaram as principais iniciativas de EC e Bioeconomia que tem sido norteadoras das ações de diversos países e apontaram para a necessidade de maior cooperação entre a comunidade científica, as empresas privadas, o governo e a sociedade para que se estabeleça uma política ambiental eficaz.
\end{abstract}

Palavras-chave: Economia circular; Bioeconomia; Biorrefinaria; Sustentabilidade; Revisão sistemática da literatura.

\section{ABSTRACT}

Given the consensus on the urgency of environmental issues and growing concern about the scarcity of natural resources, new development models have been proposed. Considering the potential contribution of the Circular Economy (CE) and Bioeconomy approaches to this context, this study sought to answer the following research question: how are the models of EC and Bioeconomy related in the literature? Based on this question, a systematic literature review was developed with the aim of analyzing: (i) how research addresses this relationship; (ii) what are the characteristics of the studies; (iii) which are the most recurrent methodological aspects; (iv) what are the main existing initiatives and programs; and (v) who are the main actors. This previous study advances knowledge about EC and Bioeconomy as the main results demonstrate the increased interest of researchers in the theme, pointed to the complementarity of approaches based on the generated keyword network, listed the main initiatives of EC and Bioeconomy that have been guiding the actions of several countries and pointed to the need for greater collaboration between the scientific community, such as private companies, the government and society, in order to establish an effective environmental policy.

Keywords: Circular Economy; Bioeconomy; Biorefinery; Sustainability; Systematic Literature Review.

Recebido em: 29-05-2020 Aceito em: 26-08-2020

\section{INTRODUÇÃO}

As últimas décadas foram marcadas pelo crescimento econômico mundial, crescimento populacional, aceleração do processo de urbanização, aumento do consumo e rapidez na circulação de informações, produtos, pessoas, capitais e serviços. As implicações ambientais desses fenômenos são de grande relevância e vem sendo objeto de reflexão não só da academia e da comunidade científica, como também de organismos internacionais, dos governos e da sociedade civil. O caráter planetário de certos problemas ambientais criou um fenômeno de interdependência crescente entre os países, resultando em regras de governança e cooperação internacional (BURSZTYN; BURSZTYN, 2012). Um exemplo disso é a Conferência das Nações Unidas sobre Mudança Climática que em seu Acordo de Paris reconhece, dentre outras medidas, que as mudanças climáticas representam uma ameaça urgente e potencialmente irreversível para as sociedades humanas e para o planeta e, portanto, requer a mais ampla cooperação possível de todos os países e sua participação numa resposta internacional eficaz e apropriada, com vista a

\footnotetext{
"Doutoranda em Desenvolvimento Sustentável (PPG-CDS). Mestre em Administração pelo PPGA/UnB. Centro de Desenvolvimento Sustentável, Universidade de Brasília. E-mail: emiliaofaria@gmail.com

"Doutor em Engenharia Mecânica pela Universidade Técnica de Lisboa. Mestre em Engenharia Química pela Universidade Federal do Rio de janeiro, Professor da Universidade de Brasília.
} 
acelerar a redução das emissões globais de gases de efeito estufa (UN, 2015).

Nesse contexto, uma das principais preocupações está relacionada com a dependência dos combustíveis fósseis associada com os níveis crescentes de dióxido de carbono $\left(\mathrm{CO}_{2}\right)$ na atmosfera, o que levou a um impulso global para o desenvolvimento de tecnologias renováveis e ecológicas (MOHAN et al., 2016; MOHAN et al., 2017). Novos modelos de desenvolvimento baseados no aumento da integração entre os sistemas econômicos e ecológicos estão surgindo em resposta a um paradigma estritamente voltado para a eficiência econômica (COLBY, 1991). Duas abordagens se apresentam promissoras em atingir esse objetivo: a Economia Circular (EC) e a Bioeconomia. Em países como a China, Estados Unidos, Itália, Dinamarca, esses conceitos já fazem parte desse discurso econômico e político associado à ideia de uma mudança necessária e iminente nos modos de produção e consumo, para preservar os recursos e o planeta (GENG et al., 2012;HAN et al., 2017; MCDOWALL et al., 2017).

A EC preconiza novas experiências de produção e consumo baseadas em um modelo econômico destinado a otimizar o uso de recursos, bem como reduzir ou eliminar o desperdício. É uma abordagem que tem atraído a comunidade empresarial uma vez que a sua lógica faz sentido do ponto de vista ambiental e comercial. Por que não aproveitar o valor econômico do recurso o máximo de vezes possível? Essa é uma das premissas básicas da EC, a transformação de um determinado subproduto da indústria em um recurso para uma segunda indústria (D'AMATO et al., 2017; KORHONEN; HONKASALO; SEPPÄLÄ, 2018). De modo análogo, a Bioeconomia fundamenta-se em uma produção de base biológica com ciclos materiais integrados e com foco em processos inovativos. A despeito de seus benefícios, a biomassa e os recursos biológicos ainda não são usados e preservados de maneira ideal (THE BIOECONOMY COUNCIL, 2015).

Em que pese os avanços na agenda do desenvolvimento sustentável e o crescente interesse acadêmico e do mercado nessas áreas, na prática, essas mudanças ainda não se concretizaram. A economia global ainda tem como modelo vigente o modo linear de produção, no qual os bens de consumo são fabricados a partir de matérias-primas, vendidos, usados e descartados como resíduos (SAAVEDRA et al., 2018).

Deste modo, torna-se imperativo ampliar a compreensão sobre esses novos modelos que priorizam o cuidado com o meio ambiente e transformam a ótica com que se observa a dinâmica econômica, uma vez que as externalidades negativas já não podem mais ser ignoradas. Partindo da premissa que cada um desses modelos valoriza certos problemas e prescreve diferentes soluções, estratégias e tecnologias, tornase desafiador definir, formular e implementar novas políticas (CEPAL, 2016).

Considerando a contribuição potencial das abordagens de EC e Bioeconomia para esse contexto de transição, coloca-se a seguinte questão de pesquisa: como os modelos de EC e Bioeconomia se relacionam na literatura? A partir dessa indagação pretende-se desenvolver uma revisão sistemática da literatura com o objetivo de analisar: (i) como as pesquisas abordam essa relação (ii) quais são as características desses estudos (distribuição temporal das publicações, principais periódicos grandes temas abordados, os autores e seus respectivos países), (iii) quais os aspectos metodológicos mais recorrentes, (iv) quais são as principais iniciativas e programas existentes, e (v) quais são os principais atores envolvidos.

A fim de endereçar essas questões, este artigo divide-se em quatro partes, além desta introdução: 1) apresenta-se os conceitos de EC e Bioeconomia que embasaram o estudo; 2) expõe-se os procedimentos metodológicos adotados para analisar e desenvolver o estudo; 3 ) apresenta-se os resultados e a discussão deles; 4) apresenta-se as considerações finais do estudo.

\section{REFERENCIAL TEÓRICO}

\subsection{Economia Circular}

O modelo de EC tem conquistado espaço entre os formuladores de políticas, influenciando governos e agências intergovernamentais em nível local, regional, nacional e internacional (ELLEN MACARTHUR FOUNDATION, 2015). A EC tem suas raízes conceituais na ecologia industrial e metabolismo industrial, que preveem uma forma de simbiose material diferente entre empresas e processos produtivos. Subjacente à abordagem da ecologia industrial está a ideia de que a circularização da economia será benéfica para a sociedade como um todo. Seus benefícios estão relacionados à redução do uso do meio ambiente como destino final de resíduos bem como a diminuição do uso de matérias primas para a atividade econômica. A abordagem vem se popularizando desde os anos 90, com a adoção de pacotes de EC pela Comissão Europeia, pelo governo da China e da Alemanha (ANDERSEN, 2007; D'AMATO et al., 2017; GEISSDOERFER et al., 2017; YUAN et al., 2006).

Em 2010, foi criada a Ellen MacArthur Foundation com a missão de acelerar a transição rumo a uma economia circular. Desde então, a fundação se articula como uma das líderes globais do pensamento, inserindo a EC na agenda de diversos stakeholders. Para eles, a EC é regenerativa e restaurativa por princípio, tendo como objetivo manter produtos, componentes e materiais em seu mais alto nível de 
utilidade e valor o tempo todo. Na concepção de seus criadores, a EC consiste em um ciclo de desenvolvimento positivo contínuo que preserva e aprimora o capital natural, otimiza a produção de recursos e minimiza riscos sistêmicos administrando estoques finitos e fluxos renováveis (ELLEN MACARTHUR FOUNDATION, 2017). De acordo com a Fundação, a EC se assenta em três princípios: (i) preservar e aumentar o capital natural controlando os estoques finitos e equilibrando os fluxos de recursos renováveis; (ii) otimizar a produção de recursos fazendo circular produtos, componentes e materiais no mais alto nível de utilidade o tempo todo, tanto no ciclo técnico quanto no biológico; e (iii) fomentar a eficácia do sistema e revelando as externalidades negativas e excluindo-as dos projetos. Além desses princípios, as características da EC são: design sem resíduo; criar resiliência através da diversidade; transitar para o uso de energia proveniente de fontes renováveis; pensar de modo sistêmico; pensar em cascatas.

Com o aumento do debate sobre as práticas de EC e sua possível adoção como modelo de negócios por parte do mercado, esforços por parte da academia foram feitos em busca de um desenvolvimento teórico mais consistente (MURRAY; SKENE; HAYNES, 2017). Nesse sentido, Geissdoerfer et al. (2017), com base em diferentes contribuições, definem a EC como um sistema regenerativo no qual a entrada de recursos e o desperdício, emissão e perda de energia são minimizados pela desaceleração, fechamento e estreitamento dos fluxos de matéria e energia. Isso pode ser alcançado por meio de um projeto duradouro, de manutenção, reparo, reutilização, remanufatura, remodelagem e reciclagem.

Adicionalmente, Murray, Skene e Haynes (2017) apontam para a importância de buscar um equilíbrio entre as dimensões social, econômica e ambiental da EC. Para eles, a EC deve ser vista como um modelo econômico em que planejamento, recursos, aquisições, produção e reprocessamento sejam projetados e gerenciados, tanto como processo, quanto resultado, a fim de maximizar o funcionamento do ecossistema e o bem-estar humano.

Nessa mesma perspectiva, Korhonen, Honkasalo e Seppala (2018) também sugerem que a definição de EC deve incorporar as três dimensões do desenvolvimento sustentável. Eles compreendem a EC como uma iniciativa do desenvolvimento sustentável que tem como objetivo reduzir os fluxos lineares de produção de materiais e energia dos sistemas de consumo e produção. Para isso, são aplicados os conceitos de ciclos de materiais, fluxos de energia renováveis e em cascata.

\subsection{Bioeconomia}

Essa mudança da visão econômica linear baseada em combustíveis fósseis para uma economia cíclica evidencia o impacto negativo da ação antrópica no meio ambiente. Para que esse impacto seja minimizado em todas as cadeias de valor, os recursos renováveis e o consumo de biomassa se tornam cada vez mais necessários (MOHAN et al., 2017; SADHUKHAN et al., 2017). Desde a origem do conhecimento biotecnológico moderno na década de 1970, com pesquisas em diversas áreas como genética, biologia molecular, bioquímica, houve um crescente interesse e expectativas pelo desenvolvimento de uma indústria de biotecnologia. Portanto, a biotecnologia tem sido caracterizada como um novo setor industrial que transformará a sociedade e a economia (BIRCH; TYFIELD, 2013). Embora, a utilização humana de materiais biogênicos ou biomassa como matéria-prima não seja algo novo, percebese, atualmente, um interesse renovado na exploração efetiva inevitável de resíduos orgânicos, provocado pelo objetivo de reduzir a pegada ecológica e alcançar um fornecimento mais seguro de recursos renováveis (MOHAN et al., 2016).

Nesse sentido, o desenvolvimento da bioeconomia, a partir da biotecnologia, está pautado em um argumento bem convincente. Acredita-se que até 2050, a população mundial deverá atingir 9 bilhões, o que colocará uma pressão sem precedentes sobre o meio ambiente e seus recursos, ou seja, as ameaças das mudanças climáticas, o esgotamento da biodiversidade, a escassez de água e terra e o aumento dos níveis de poluição, necessitam de novas soluções. Portanto, uma bioeconomia orientada pela inovação, que tem como objetivo final a conservação dos recursos naturais, pode fornecer renovação, circularidade e multifuncionalidade, criando empregos, crescimento e prosperidade nas áreas rurais, costeiras e urbanas. Sendo assim, a bioeconomia promete liderar a próxima onda emergente do desenvolvimento econômico global, tendo como base a energia renovável, matérias-primas renováveis, produtos recicláveis, processos biológicos, organismos naturais ou geneticamente modificados, fermentação, biotecnologia e biologia molecular (BELL et al., 2017; DUPONT-INGLIS; BORG, 2017; MCCORMICK; KAUTTO, 2013; SZÉKÁCS, 2017).

Em uma tentativa de definir o conceito de Bioeconomia no âmbito europeu, Mccormick e Kautto (2013) a definem como uma economia em que as partes básicas da composição de materiais, produtos químicos e energia são derivados de recursos biológicos renováveis. Para os autores, a biotecnologia e a biorrefinaria são os componentes essenciais da bioeconomia.

Em outro estudo, a partir de uma revisão da literatura, Bugge, Hansen e Klitkou (2016) identificaram três visões da Bioeconomia. A primeira é a visão da biotecnologia que enfatiza a importância da pesquisa, 
da aplicação e comercialização da biotecnologia em diferentes setores da economia. A segunda visão é a dos biorrecursos que se concentra no processamento e atualização de matérias-primas biológicas, bem como no estabelecimento de novas cadeias de valor. Por último, a visão da bioecologia destaca a sustentabilidade e os processos ecológicos que otimizam o uso de energia e nutrientes, promovem a biodiversidade e evitam monoculturas e degradação do solo.

No âmbito das políticas públicas, em 2017, a estratégia de bioeconomia da União Europeia completou cinco anos. A proposta inicial surgiu como uma forma de avançar para uma sociedade mais sustentável, com o argumento de que a obtenção de crescimento e emprego na Europa só poderia ser alcançada por meio do uso eficiente dos recursos, criando uma economia circular capaz de reduzir a geração de resíduos e fazer uso de resíduos como recurso. A Bioeconomia europeia já é um importante setor econômico que representa cerca de 18 milhões de empregos em diversas indústrias bem estabelecidas. Muitos êxitos foram alcançados e há uma crescente compreensão e apreciação do papel que a bioeconomia desempenhará na entrega de um futuro mais inteligente e sustentável para a Europa e seus cidadãos (BELL et al., 2017; DUPONT-INGLIS; BORG, 2017).

Permeando os conceitos de EC e Bioeconomia, as biorrefinarias surgem como uma atividade importante para reverter a degradação ambiental. $\mathrm{O}$ conceito de produzir produtos a partir de commodities agrícolas (ou seja, biomassa) não é recente, embora, o uso da biomassa como entrada para produzir múltiplos produtos usando métodos de processamento complexos, com uma abordagem análoga a uma refinaria de petróleo é relativamente atual. A biomassa consiste em carboidratos, lignina, proteínas, gorduras e, em menor grau, vários outros produtos químicos, como vitaminas, corantes e aromas. Mais especificamente, o termo biorrefinaria refere-se à conversão de matéria-prima de biomassa em uma série de produtos químicos e energia valiosos com o mínimo de resíduos e emissões (DEMIRBAS, 2009; FERNANDO et al., 2006).

O conceito de biorrefinaria é uma aplicação técnica do princípio de EC em que a exploração da biomassa é melhorada. Na biorrefinaria, as etapas individuais da cadeia de valor imitam a exploração gradual da biomassa, a fim de explorar todos os compostos disponíveis. Se todo o recurso for utilizado, a produção de resíduo zero possivelmente será alcançada (SEGHETTA et al., 2016).

Recentemente, surgiram outros tipos de biorrefinarias, capazes de fermentar uma grande variedade de matérias-primas, dependendo da estação e da origem da biomassa. Estes variam desde resíduos agrícolas e florestais até resíduos sólidos municipais. Estes tipos de biorrefinaria, conhecidos como biorrefinarias de segunda geração, podem usar uma ampla gama e uma mistura de matérias-primas para a fermentação, como a celulose, hemicelulose, lignina entre outros. Já a biorrefinaria de terceira geração, ainda em fase de desenvolvimento, é baseada no cultivo e na colheita de microalgas, seguida da extração de óleo e sua posterior transformação em biodiesel. A vantagem do uso de microalgas como matéria-prima é seu potencial de usar fluxos ricos em CO2 e luz solar como fonte de energia (DEMIRBAS, 2009; EGEA et al., 2017).

\section{ASPECTOS METODOLÓGICOS}

Para a consecução do objetivo do estudo, foi realizada uma revisão sistemática da literatura sobre os conceitos de EC e Bioeconomia. A escolha por esse método se justifica pelo fato de que diferentemente da revisão de literatura tradicional, o objetivo de uma revisão sistemática consiste em fornecer uma lista tão completa quanto possível de todos os estudos publicados a um determinado assunto, utilizando critérios explícitos e rigorosos (CRONIN et al., 2008). Portanto, este estudo baseou-se no procedimento de revisão sistemática proposto por Cronin et al. (2008), já utilizado por Guarnieri et al. (2015). Com o objetivo de conferir fiabilidade à revisão, Cronin et al. (2008) propõem um protocolo a ser seguido pelos pesquisadores, a saber:

(a) Formulação da questão norteadora de pesquisa: como os modelos de EC e Bioeconomia se relacionam na literatura?;

(b) estabelecimento de critérios de inclusão e exclusão: optou-se pela base de dados Scopus por ser a maior base de dados de citações e resumos de literatura revisada por pares; optou-se por apenas artigos científicos publicados em revistas sem definição de critério temporal; em relação às palavras-chave, foram utilizadas duas combinações: "Circular Economy" and "Bioeconomy" e "Circular Economy", "Bioeconomy" and "Biorefinery". A busca ocorreu no título, no resumo e nas palavras-chave; quanto aos operadores booleanos utilizou-se somente $o$ and;

(c) seleção e acesso de literatura: a primeira combinação retornou 26 resultados; já a segunda combinação retornou 13. Ao fazer a comparação dos resultados, constatou-se que os 13 trabalhos da segunda busca estavam contidos na primeira combinação. Dos 26 resultados, três eram capítulos de livro e um eram notas de uma palestra. Portanto, seguindo os critérios de inclusão e exclusão mencionados no tópico anterior, quatro trabalhos foram excluídos; 
(d) avaliação da qualidade da literatura incluída na revisão: foi realizada uma análise do resumo dos artigos, de modo a verificar a pertinência e qualidade dos artigos selecionados. Feita a análise, 20 artigos foram mantidos e

(e) análise, síntese e disseminação dos resultados: por fim, os artigos foram analisados detalhadamente.

\section{RESULTADOS E DISCUSSÃO}

Esta seção apresenta os resultados dos artigos selecionados para a análise, considerando-se o protocolo de revisão sistemática estabelecido na seção 3. Os primeiros estudos encontrados a partir das combinações desejadas dataram de 2016, demonstrando a recenticidade das pesquisas englobando os dois termos. Apesar disso, é patente o aumento do interesse dos pesquisadores quanto ao tema, uma vez que em 2017, esse número aumentou em 133\%. Em 2016 foram 6 artigos publicados, enquanto em 2017 foram 14.

No que tange aos países de origem dos autores dos estudos (gráfico 1), em primeiro lugar estão a Bélgica e os Estados Unidos da América, com cinco autores. Em segundo lugar estão a Índia e a Alemanha com quatro pesquisadores. Os demais países contabilizaram três (Malásia e Espanha), dois (Dinamarca, Finlândia, França, Irlanda e Reino Unido) ou apenas um autor (Grécia, Hungria, Itália e Taiwan). Percebe-se sobremaneira um grande interesse dos países europeus sobre o assunto. Essas informações vão de encontro às iniciativas recentes de EC e Bioeconomia formuladas pela União Europeia em 2012 e 2015, além do Plano Nacional de Bioeconomia dos Estados Unidos de 2012 (D’AMATO et al., 2017).

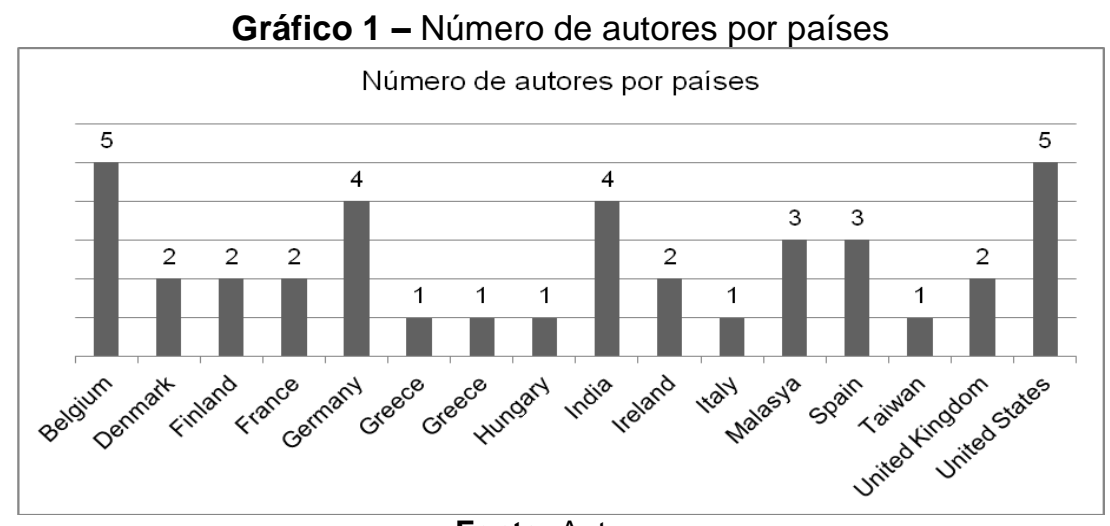

Fonte: Autores

Quanto aos periódicos que serviram de veículos para a publicação dos artigos (gráfico 2), a revista New Biotechnology e o Journal of Cleaner Production destacaram-se ao publicarem seis e cinco artigos respectivamente. Os demais artigos foram publicados em diferentes periódicos. Ao analisar os principais temas emergentes, nota-se que o principal assunto diz respeito à Ciência Ambiental, desvelando que, embora o tema tenha merecido a atenção de outras áreas como Economia e Gestão, Ciência Ambiental ainda é a área que domina as investigações sobre EC e Bioeconomia.

Gráfico 2 - Revistas que publicaram sobre o tema

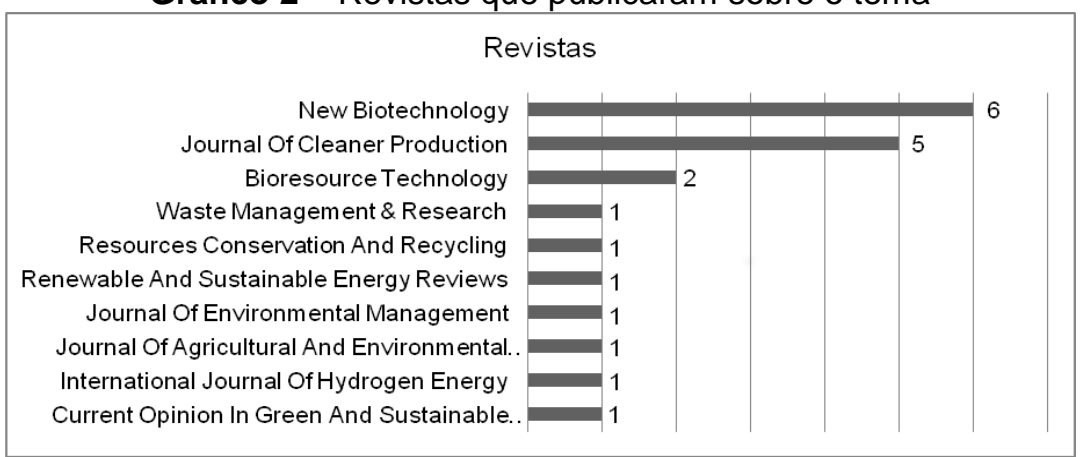

Fonte: Autores

$\mathrm{Na}$ figura 1, dois indicadores bibliométricos foram utilizados para analisar e discutir a rede criada pelas palavras-chave: o número de ocorrências e a relação entre os nós (cada nó é uma palavra-chave). Quanto maior o número de conexões, maior a força do nó na rede e maior centralidade. Observa-se que há 
uma maior centralidade e força ao redor do termo EC, sendo que a segunda palavra-chave com maior destaque é Bioeconomia, o que reforça a relação de complementaridade das abordagens. Os princípios da EC se concentram em preservar e aprimorar o capital natural, otimizar os recursos e estimular a efetividade do sistema, ou seja, é uma abordagem holística que se adapta a qualquer escala, por isso o termo se liga a tantos outros, como pode ser visto na figura 1 (ELLEN MACARTHUR FOUNDATION, 2015). Nesse sentido, a Bioeconomia se mostra instrumental para a implementação da EC na medida em que ela integra a produção de recursos renováveis, regenera $\mathrm{CO} 2$ e usa matéria-prima renovável para fazer produtos de uso diário mais verdes (CARREZ; LEEUWEN, VAN, 2015).

$\mathrm{Na}$ figura 1, há ainda termos correlatos que estão mais dispersos, o que indica menor incidência de estudos relacionados aos temas. A análise dessa rede revela a existência de três clusters. $\mathrm{O}$ cluster vermelho relaciona os termos: objetivos do Desenvolvimento Sustentável, Comissão Europeia, biorrefinaria, biomassa e EC. O cluster azul conecta os termos Bioeconomia, sustentabilidade e economia verde. O terceiro cluster engloba os termos bioprodutos, biocombustíveis, avaliação do ciclo de vida, gestão de resíduos e biorrefinaria de resíduos.

Figura 1 - Rede de palavras-chave

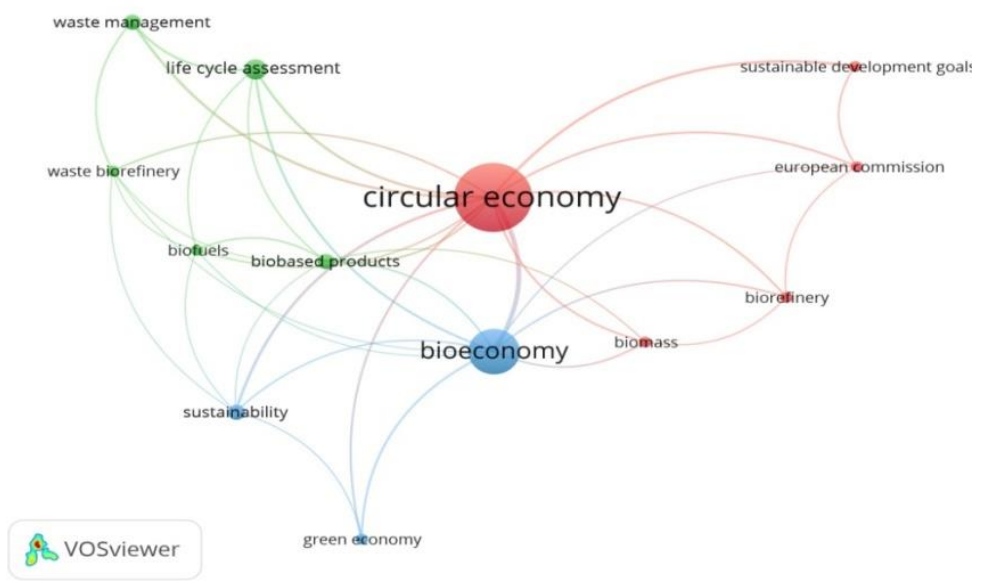

Fonte: Autores

As escalas de análise dos estudos variam em tempo e espaço. Os estudos de Mohan et al. (2016) e D'Amato et al. (2017) tratam as questões relacionadas à EC e à Bioeconomia a nível global explorando a integração dos diferentes modelos como caminhos para o desenvolvimento e a sustentabilidade. Por outro lado, os estudos de Bell et al. (2017), Mengal et al. (2017), Maina et al. (2017) e Dupont-Inglis e Borg (2017) tratam os temas a nível regional da União Europeia. Os demais estudos se restringem a análises locais por países, quais sejam: Malásia (SADHUKHAN et al., 2017); Irlanda (Oldfield et al., 2016); Dinamarca (SEGHETTA et al., 2016); Espanha (Egea et al., 2017; Lainez et al., 2016; Serrano et al., 2017); Índia (Mohan et al., 2017); Estados Unidos (OLDFIELD et al., 2017) e Itália (MAINA; KACHRIMANIDOU; KOUTINAS, 2017).

Dentre os métodos de análise utilizados (tabela 1), percebe-se um domínio do método LCA ou ACV (Life Cicle Assessement ou Avaliação do Ciclo de Vida) nas pesquisas que tratam dos impactos ambientais dos processos produtivos. O ACV se apresenta como uma das principais ferramentas de diagnóstico dos benefícios ambientais de diversas práticas, sendo capaz de analisar sistemas circulares, sistemas de reciclagem e sistemas produto-serviço (DADDI; NUCCI; IRALDO, 2017;SCHEEPENS; VOGTLÄNDER; BREZET, 2016). Sadhukhan et al. (2017) fizeram o uso do método LCA combinado a técnicas econométricas a fim de compor indicadores quantitativos e qualitativos. Por sua vez, Lee (2017) comparou nove modelos econométricos que medem a eficiência de diferentes gerações de energia no intuito de apontar o modelo econométrico mais adequado para avaliar os impactos numéricos da $E C$, da bioeconomia e bioenergia. $O$ resultado da pesquisa apontou para o modelo de regressão e o modelo de séries temporais como mais pertinente para esse tipo de análise. Cabe ressaltar que muitos estudos não tiveram um método de análise definido por se tratarem de artigos teóricos conceituais. 
Tabela 1 - Síntese dos principais métodos de análise

\begin{tabular}{l|l}
\hline Autores & Métodos \\
\hline (Sadhukhan et al., 2017) & $\begin{array}{l}\text { Técnicas econométricas e LCA (indicadores } \\
\text { quantitativos e qualitativos) }\end{array}$ \\
\hline (Hildebrandt et al., 2017; Oldfield et al., 2017, & $\begin{array}{l}\text { LCA (Life Cicle Assessement) - Análise do } \\
\text { Ciclo de Vida }\end{array}$ \\
\hline (Pitkänen et al., 2016) & \begin{tabular}{l} 
Estudos de casos comparados \\
\hline (Lee, 2017)
\end{tabular} \\
$\begin{array}{l}\text { Modelo de regressão e modelo de séries } \\
\text { temporais }\end{array}$ \\
\hline
\end{tabular}

Fonte: Autores

No que diz respeito aos programas e iniciativas já existentes, percebe-se um crescimento dessas práticas calcadas no respeito ao meio ambiente e na redução da dependência de recursos como os combustíveis fósseis, com destaque para as políticas europeias que empregam recursos e investimentos em pesquisa, desenvolvimento e treinamento nessas áreas. Algumas dessas iniciativas já estão mais consolidadas como a Estratégia Europeia de Bioeconomia avaliada nos estudos de Mengal et al. (2017) e Bell et al. (2017), outras ainda estão em processo de construção e debate como o Plano da Malásia (20162020) apresentado por Sadhukhan et al. (2017) e o Plano espanhol de Bioeconomia (2030) exposto por Lainez et al. (2016).

A Tabela 2 sintetiza as informações a respeito dos programas e iniciativas mapeados nos estudos

Tabela 2 - Síntese dos principais programas e iniciativas

\begin{tabular}{|c|c|}
\hline Autores & Iniciativas \\
\hline (SADHUKHAN et al., 2017) & Plano de desenvolvimento da Malásia (2016-2020); \\
\hline (SEGHETTA et al., 2016) & Projeto de Biorrefinaria de Macroalgas; \\
\hline (PITKÄNEN et al., 2016) & $\begin{array}{l}\text { Programa Nacional de Revolução Energética (DE); } \\
\text { Programa Nacional de uso de madeira para construção (FI); } \\
\text { BIODECOL2 - projeto para implantação do biogás (FR); } \\
\text { Projeto para minimizar os resíduos orgânicos municipais (FR); } \\
\text { Projeto para desenvolver um modelo de cidade sustentável (FI); } \\
\text { HINKU - rede de municípios finlandeses com o objetivo de criar e } \\
\text { implementar soluções para reduzir as emissões de gases de efeito } \\
\text { estufa (FI); } \\
\text { Certificação para reduções de emissões no mercado voluntário de } \\
\text { carbono (DE); } \\
\text { Simbiose Industrial em Dunkirk (FR); } \\
\text { Healthy Sand (NL) - estratégias específicas de manejo de fazendas } \\
\text { que aumentariam os níveis de matéria orgânica do solo (NL); } \\
\text { MAB3 - Biorrefinaria de Macroalgas (DK); }\end{array}$ \\
\hline (LAINEZ et al., 2016) & $\begin{array}{l}\text { Estratégia espanhola de bioeconomia } 2030 \text { e Plano de ação de } \\
\text { bioeconomia } 2016 \text { ambos da Secretaria de Estado, de Pesquisa, } \\
\text { Desenvolvimento e Inovação; }\end{array}$ \\
\hline (BELL et al., 2017) & $\begin{array}{l}\text { Estratégia Europeia de Bioeconomia (Bio-based Industries Joint } \\
\text { Undertaking); }\end{array}$ \\
\hline (OLDFIELD et al., 2017) & $\begin{array}{l}\text { Projetos inovadores para formulação de pesticidas para atender a } \\
\text { demanda global de controle de pragas na agricultura; }\end{array}$ \\
\hline (SOMMERHUBER et al., 2017) & Programa Alemão de Eficiência de Recursos (ProgRess) II; \\
\hline (MENGAL et al., 2017) & $\begin{array}{l}\text { BBI JU - parceria público-privada (PPP) entre a Comissão Europeia } \\
(\mathrm{CE}) \text { e o Consórcio das Indústrias de biotecnologia (BIC); }\end{array}$ \\
\hline (SERRANO et al., 2017) & $\begin{array}{l}\text { Proposta de digestão anaeróbica como método de gerenciamento } \\
\text { adequado para resíduos sólidos; }\end{array}$ \\
\hline $\begin{array}{l}\text { (MAINA; } \quad \text { KACHRIMANIDOU; } \\
\text { KOUTINAS, 2017) }\end{array}$ & $\begin{array}{l}\text { Conversão de uma refinaria convencional em Porto Marghera } \\
\text { (Veneza, Itália) em uma biorrefinaria através da tecnologia ecofining; }\end{array}$ \\
\hline $\begin{array}{l}\text { (DUPONT-INGLIS; } \quad \text { BORG, } \\
\text { 2017) }\end{array}$ & $\begin{array}{l}\text { BIO-TIC. Programa para impulsionar o setor de biotecnologia na } \\
\text { Europa (2015); }\end{array}$ \\
\hline (MOHAN et al., 2017) & $\begin{array}{l}\text { Criação de um ministério na Índia para coordenar esforços na área } \\
\text { industrial e de biotecnologia. }\end{array}$ \\
\hline
\end{tabular}

Fonte: Autores 
Para além dos programas e iniciativas já descritos, à medida que os conceitos de EC e Bioeconomia ganham notoriedade e impulso, eles tornam-se influentes para vários stakeholders como forma de apoiar ou legitimar seus interesses e atividades (D'AMATO et al., 2017).

Os estudos de Lainez et al. (2016), Bell et al. (2017), Egea et al. (2017), Oldfield et al. (2017), Mengal et al. (2017), Dupont-Inglis e Borg (2017) e Mohan et al. (2017) apontam para a necessidade de cooperação entre a comunidade científica, as empresas privadas, o governo e a sociedade para que se estabeleça uma política ambiental eficaz baseada nos princípios da Bioeconomia e da EC. Diversos setores econômicos já reconhecem a biotecnologia industrial como uma das principais forças tecnológicas da União Europeia, sendo hoje reconhecida como uma tecnologia chave para permitir uma bioeconomia mais competitiva e sustentável (DUPONT-INGLIS; BORG, 2017). Nesse sentido, (BEZAMA, 2018) advoga que é necessário um espectro mais amplo de atores que inclua não só as instituições envolvidas no desenvolvimento de tecnologia, como também os atores do mercado e da sociedade que são os responsáveis por gerar a demanda por novos processos e produtos.

Diante desse contexto, estudos como Oldfield, White, e Holden (2016), Seghetta et al. (2016), Egea et al. (2017), Sadhukhan et al. (2017), Oldfield et al. (2017), Hildebrandt et al. (2017), Sommerhuber et al. (2017) e Serrano et al. (2017) mostram que as empresas dos mais diversos ramos de atuação, desde o setor industrial ao setor agrícola, estão investindo em pesquisa e inovação a fim de acompanhar essa evolução. O bussiness-as-usual já não se enquadra nas demandas da produção atual.

Por fim, as biorrefinarias aparecem nos estudos como uma opção verde sustentável para utilizar os resíduos e produzir uma gama de bioprodutos e biocombustíveis comercializáveis em relação à refinaria petroquímica. Elas são altamente eficientes em termos de energia e utilizam processos produtivos com resíduo zero, permitindo que as indústrias fabriquem produtos amigáveis com o meio ambiente com pequenas pegadas de carbono e água (EGEA et al., 2017; MOHAN et al., 2016).

Ainda nessa perspectiva, os estudos de Seghetta et al. (2016) e Lainez et al. (2016) apontam para o potencial das biorrefinarias de micro e macroalgas que se enquadram no conceito de bioeconomia azul. Os estudos mostram que as biorrefinarias de macro/microalgas (MAB) podem contribuir para uma EC regenerativa por meio da restauração ambiental e da mitigação das mudanças climáticas.

Portanto, para Mohan et al. (2016) faz-se necessária a avaliação dos processos produtivos como uma estratégia integrada de modo que os modelos propostos abordem a visão da EC de ciclo fechado no qual os resíduos são valorizados por meio de vários processos biotecnológicos. Nessa mesma linha, Maina et al. (2017) acreditam que essas abordagens integradas e holísticas de utilização de resíduos orgânicos como matérias-primas industriais impulsionarão a transição para a era da bioeconomia.

\section{CONCLUSÃO}

Os estudos analisados a partir das combinações propostas entre os conceitos de Bioeconomia e EC indicam que se trata de fenômenos recentes e de crescente interesse dos pesquisadores. $O$ método utilizado de revisão sistemática de literatura permitiu a compilação de evidências sobre o surgimento de uma nova abordagem que questiona a base econômica baseada na trajetória linear de extrair, transformar, consumir e descartar. Além disso, o estudo também esclareceu conceitos e práticas, identificou os principais periódicos, grandes áreas e países que tem se debruçado sobre o assunto.

Quanto à análise das palavras-chave, percebeu-se que a EC já se apresenta como framework consolidado nas pesquisas recentes, tendo sido a palavra-chave mais recorrente e mais dominante na rede. A Bioeconomia surge como a segunda palavra-chave mais relevante, com forte conexão com o termo EC, determinando importantes ligações com outras palavras-chaves como biorrefinaria, objetivos do Desenvolvimento Sustentável e Economia Verde. Essa relação apontada pela literatura solidifica a ideia de que o desenvolvimento do setor da economia baseado no uso sustentável de recursos e processos biotecnológicos, a bioeconomia, deve ser um dos pilares da economia circular, uma vez que ela busca maximizar a utilização de produtos biológicos, minimizar o desperdício da cadeia produtiva. Princípios esses que coadunam com o projeto proposto pela $\mathrm{EC}$, de manutenção, reparo, reutilização, remanufatura, remodelagem e reciclagem.

Portanto, diante dos resultados da pesquisa, o panorama que por ora se configura indica o aumento de programas e iniciativas de EC e Bioeconomia pelo mundo, bem como do uso das biorrefinarias nesse contexto e ratifica seu caráter contemporâneo. Entretanto, apesar do crescimento significativo desses conceitos, várias controvérsias permanecem em voga, considerando questões tecnológicas, sociais e de avaliação. Sendo assim, sugere-se uma agenda de pesquisa que inclua as seguintes questões: 0 arcabouço legal necessário para regular o uso dos biorrecursos; estudos que avaliem a toxicidade dos subprodutos dos processos produtivos; esforços no estabelecimento de padrões para os produtos obtidos nas biorrefinarias; estudos sobre os potenciais impactos negativos da bioeconomia relacionados a agricultura e a produção de alimentos; avaliações relativas as alterações causadas nos recursos naturais e 
na biodiversidade do ecossistema devido ao uso de diferentes tecnologias; desenvolvimento de indicadores de desempenho para a Bioeconomia; e análise do custo-benefício das biorrefinarias (CARUS; DAMMER, 2018; FERNANDO et al., 2006; MCCORMICK; KAUTTO, 2013; MOHAN, S.V. et al., 2016).

\section{REFERÊNCIAS}

ANDERSEN, M. S. An introductory note on the environmental economics of the circular economy. Sustainability Science, [s.l.], v. 2, no 1, p. 133-140, 2007. ISBN: 1862-4065, ISSN: 18624065, DOI: 10.1007/s11625-006-0013-6.

$\mathrm{BELL}, \mathrm{J}$. et al. EU ambition to build the world's leading bioeconomy-Uncertain times demand innovative and sustainable solutions. New Biotechnology, European Commission, Brussels, Belgium, v. 40, p. 25-30, 2017. ISSN: 18764347, DOI: 10.1016/j.nbt. 2017.06.010.

BEZAMA, A. Understanding the systems that characterise the circular economy and the bioeconomy. Waste Management and Research, [s.I.], v. 36, no 7, p. 553-554, 2018. ISSN: 10963669, DOI: 10.1177/0734242X18787954.

BIRCH, K.; TYFIELD, D. Theorizing the Bioeconomy. Science, Technology, \& Human Values, [s.I.], v. 38, nº 3, p. 299327, 2013. ISSN: 0162-2439, DOI: 10.1177/0162243912442398.

BUGGE, M. M.; HANSEN, T.; KLITKOU, A. What is the bioeconomy? A review of the literature. Sustainability (Switzerland), [s.I.], v. 8, no 7, 2016. ISSN: 20711050, DOI: 10.3390/su8070691.

BURSZTYN, M.; BURSZTYN, M. A. Fundamentos de Política e Gestão Ambiental. Rio de Janeiro: Garamond, 2012. 612 p. ISBN: 9788576172901.

CARREZ, D.; LEEUWEN, P. VAN. Bioeconomy: circular by nature. The European Flles, [s.I.], no 38, p. 34-35, 2015.

CARUS, M.; DAMMER, L. The Circular Bioeconomy - Concepts, Opportunities, and Limitations. Industrial Biotechnology, [s.I.], v. 14, no 2, p. 83-91, 2018. ISSN: 15509087, DOI: 10.1089/ind.2018.29121.mca.

CEPAL. Horizontes 2030 a igualdade no centro do desenvolvimento sustentável. CEPAL (Org.). In: Trigésimo sexto período de sessões da CEPAL. Cidade do México: [s.n.], 2016.

COLBY, M. E. Environmental management in development: the evolution of paradigms. Ecological Economics, [s.I.], v. 3, no 3, p. 193-213, 1991. ISBN: 0921-8009, ISSN: 09218009, DOI: 10.1016/0921-8009(91)90032-A.

CRONIN, P.; RYAN, F.; COUGHLAN, M. Undertaking a literature review: A step-by-step approach. British Journal of Nursing, [s.I.], v. 17, p. 38-43, 2008.

D'AMATO, D. et al. Green, circular, bio economy: A comparative analysis of sustainability avenues. Journal of Cleaner Production, [s.I.], v. 168, p. 716-734, 2017. ISSN: 09596526, DOI: 10.1016/j.jclepro.2017.09.053.

DADDI, T.; NUCCI, B.; IRALDO, F. Using Life Cycle Assessment (LCA) to measure the environmental benefits of industrial symbiosis in an industrial cluster of SMEs. Journal of Cleaner Production, Sant'Anna School of Advanced Studies - Institute of Management, Piazza Martiri della Libertà 33, Pisa, Italy, v. 147, p. 157-164, 2017. ISBN: 09596526, ISSN: 09596526, DOI: 10.1016/j.jclepro.2017.01.090.

DEMIRBAS, A. Biorefineries: Current activities and future developments. Energy Conversion and Management, [s.I.], v. 50, no 11, p. 2782-2801, 2009. ISBN: 0196-8904, ISSN: 01968904, DOI: 10.1016/j.enconman.2009.06.035.

EGEA, F. J.; TORRENTE, R. G.; AGUILAR, A. An efficient agro-industrial complex in Almería (Spain): Towards an integrated and sustainable bioeconomy model. New Biotechnology, Andalusian Centre for the Assessment and Monitoring of Global Change, Dep. of Chemistry and Physics, University of Almería, Almería, 04021, Spain, v. 6784, n 16, p. 103-112, 2017. ISSN: 18764347, DOI: 10.1016/j.nbt.2017.06.009.

ELLEN MACARTHUR FOUNDATION. Rumo À Economia Circular: O Racional De Acelerar a Transição. In: Ellen MacArthur Foundation. [s.l.]: [s.n.], 2015.

FERNANDO, S. et al. Biorefineries: Current Status, Challenges, and Future Direction. Energy Fuels, [s.I.], v. 20, nº 4, p. 1727-1737, 2006. ISBN: 0887-0624, ISSN: 0887-0624, DOI: 10.1021/ef060097w.

GEISSDOERFER, M. et al. The Circular Economy - A new sustainability paradigm? Journal of Cleaner Production, [s.l.], v. 143, p. 757-768, 2017. ISBN: 0959-6526, ISSN: 09596526, DOI: 10.1016/j.jclepro.2016.12.048.

GENG, Y. et al. Towards a national circular economy indicator system in China: An evaluation and critical analysis. Journal of Cleaner Production, Key Lab on Pollution Ecology and Environmental Engineering, Institute of Applied Ecology, Chinese Academy of Sciences, Shenyang, Liaoning Province 110016, China, v. 23, no 1, p. 216-224, 2012. ISSN: 09596526 (ISSN), DOI: 10.1016/j.jclepro.2011.07.005.

GUARNIERI, P. et al. The challenge of selecting and evaluating third-party reverse logistics providers in a multicriteria perspective: A Brazilian case. Journal of Cleaner Production, [s.l.], v. 96, p. 209-219, 2015. ISBN: 09596526 (ISSN), ISSN: 09596526, DOI: 10.1016/j.jclepro.2014.05.040.

HAN, F. et al. Circular economy measures that boost the upgrade of an aluminum industrial park. Journal of Cleaner Production, School of Environmental Science and Engineering, Shandong University, No. 27 South Shanda Road, Jinan, China, v. 168, p. 1289-1296, 2017. ISSN: 09596526 (ISSN), DOI: 10.1016/j.jclepro.2017.09.115.

HILDEBRANDT, J.; BEZAMA, A.; THRÄN, D. Cascade use indicators for selected biopolymers: Are we aiming for the right solutions in the design for recycling of bio-based polymers? Waste Management \& Research, [s.I.], v. 35, no 4, p. 367-378, 2017. ISSN: 0734-242X, DOI: 10.1177/0734242X16683445. 
KORHONEN, J.; HONKASALO, A.; SEPPÄLÄ, J. Circular Economy: The Concept and its Limitations. Ecological Economics, [s.I.], v. 143, p. 37-46, 2018. ISSN: 09218009, DOI: 10.1016/j.ecolecon.2017.06.041.

LAINEZ, M. et al. Spanish strategy on bioeconomy: Towards a knowledge based sustainable innovation. New Biotechnology, National Institute for the Agricultural and Food Research and Technology, INIA, Apdo. 8.111, Madrid, 28080, Spain, v. 40, p. 87-95, 2016. ISBN: 1871-6784, ISSN: 18764347, DOI: 10.1016/j.nbt.2017.05.006.

LEE, D. H. Econometric assessment of bioenergy development. International Journal of Hydrogen Energy, [s.I.], p. 1-17, 2017. ISSN: 03603199, DOI: 10.1016/j.jhydene.2017.08.055.

MCCORMICK, K.; KAUTTO, N. The Bioeconomy in Europe: An Overview. Sustainability (Switzerland), [s.I.], v. 5, $\mathrm{n}^{\circ}$ 6, p. 2589-2608, 2013. ISBN: 2071-1050, ISSN: 20711050, DOI: 10.3390/su5062589.

MCDOWALL, W. et al. Circular Economy Policies in China and Europe. Journal of Industrial Ecology, [s.I.], v. 21, n ${ }^{\circ}$ 3, p. 651-661, 2017. ISBN: 10881980 (ISSN), ISSN: 15309290, DOI: 10.1111/jiec.12597.

MENGAL, P. et al. Bio-based Industries Joint Undertaking: The catalyst for sustainable bio-based economic growth in Europe. New Biotechnology, Executive Director, Bio-Based Industries Joint Undertaking (BBI JU), Belgium, v. 40, p. 3139, 2017. ISSN: 18764347, DOI: 10.1016/j.nbt.2017.06.002.

MOHAN, S.V. et al. Waste biorefinery models towards sustainable circular bioeconomy: Critical review and future perspectives. Bioresource Technology, [s.I.], v. 215, p. 2-12, 2016. ISBN: 1873-2976 (Electronic)r0960-8524 (Linking), ISSN: 18732976, DOI: 10.1016/j.biortech.2016.03.130.

MOHAN, S Venkata et al. Waste derived bioeconomy in India: A perspective. New Biotechnology, [s.I.], v. 40, p. 6069, 2017. DOI: 10.1016/j.nbt.2017.06.006.

MURRAY, A.; SKENE, K.; HAYNES, K. The Circular Economy: An Interdisciplinary Exploration of the Concept and Application in a Global Context. Journal of Business Ethics, [s.I.], v. 140, n 3, p. 369-380, 2017. ISBN: 0167-4544r15730697, ISSN: 15730697, DOI: 10.1007/s10551-015-2693-2.

OLDFIELD, T. L. et al. A life cycle assessment of biosolarization as a valorization pathway for tomato pomace utilization in California. Journal of Cleaner Production, [s.I.], v. 141, p. 146-156, 2017. ISSN: 09596526, DOI: 10.1016/j.jclepro.2016.09.051.

OLDFIELD, T. L.; WHITE, E.; HOLDEN, N. M. An environmental analysis of options for utilising wasted food and food residue. Journal of Environmental Management, [s.I.], v. 183, p. 826-835, 2016. ISBN: 0301-4797, ISSN: 10958630, DOI: 10.1016/j.jenvman.2016.09.035.

PITKÄNEN, K. et al. What can be learned from practical cases of green economy? - studies from five European countries. Journal of Cleaner Production, [s.I.], v. 139, p. 666-676, 2016. ISBN: 09596526, ISSN: 09596526, DOI: 10.1016/j.jclepro.2016.08.071.

SAAVEDRA, Y. M. B. B. et al. Theoretical contribution of industrial ecology to circular economy. Journal of Cleaner Production, Department of Production Engineering, School of Engineering of São Carlos, University of São Paulo, Brazil, v. 170, p. 1514-1522, 2018. ISSN: 09596526, DOI: 10.1016/j.jclepro.2017.09.260.

SADHUKHAN, J. et al. Role of bioenergy, biorefinery and bioeconomy in sustainable development: Strategic pathways for Malaysia. Renewable and Sustainable Energy Reviews, [s.I.], nº June, 2017. ISBN: 13640321 (ISSN), ISSN: 18790690, DOI: 10.1016/j.rser.2017.06.007.

SCHEEPENS, A. E.; VOGTLÄNDER, J. G.; BREZET, J. C. Two life cycle assessment (LCA) based methods to analyse and design complex (regional) circular economy systems. Case: Making water tourism more sustainable. Journal of Cleaner Production, [s.I.], v. 114, p. 257-268, 2016. ISBN: 0959-6526, ISSN: 09596526, DOI: 10.1016/j.jclepro.2015.05.075.

SEGHETTA, M. et al. Life cycle assessment of macroalgal biorefinery for the production of ethanol, proteins and fertilizers - A step towards a regenerative bioeconomy. Journal of Cleaner Production, [s.I.], v. 137, p. 1158-1169, 2016. ISSN: 09596526, DOI: 10.1016/j.jclepro.2016.07.195.

SERRANO, A. et al. Olive mill solid waste biorefinery: High-temperature thermal pre-treatment for phenol recovery and biomethanization. Journal of Cleaner Production, [s.I.], v. 148, p. 314-323, 2017. ISSN: 09596526, DOI: 10.1016/j.jclepro.2017.01.152.

SOMMERHUBER, P. F. et al. Life cycle assessment of wood-plastic composites: Analysing alternative materials and identifying an environmental sound end-of-life option. Resources, Conservation and Recycling, [s.I.], v. 117, p. 235-248, 2017. ISBN: 0921-3449, ISSN: 18790658, DOI: 10.1016/j.resconrec.2016.10.012.

SZÉKÁCS, A. Environmental and Ecological Aspects in the Overall Assessment of Bioeconomy. Journal of Agricultural and Environmental Ethics, [s.I.], v. 30, no 1, p. 153-170, 2017. ISSN: 1573322X, DOI: 10.1007/s10806-0179651-1.

THE BIOECONOMY COUNCIL. Making Bioeconomy work for Sustainable Development. [s.l.]: [s.n.], 2015. Disponível em: <https://gbs2015.com/fileadmin/gbs2015/Downloads/Communique_final.pdf\%0Ahttp://gbs2015.com/fileadmin/gbs2015/D ownloads/Communique_final_neu.pdf>.

YUAN, Z. et al. The Circular Economy: A New Development Strategy in China. Journal of Industrial Ecology, [s.I.], v. $10, n^{\circ} 1,2$, p. 4-8, 2006. ISBN: 1530-9290, ISSN: 10881980, DOI: 10.1162/108819806775545321. 